\title{
Paediatric oncology in the Queen Elizabeth Central Hospital, Blantyre
}

\section{Trijn Israels, Kondwani Banda, Elizabeth M Molyneux}

Paediatric Department, College of Medicine, Queen Elizabeth Central Hospital, Box 360, Blantyre Malawi

Corresponding author: Prof. EM Molyneux email: emolyneux@malawi. net

\section{Introduction}

In a developing country, where infection, malnutrition and HIV infection cause an enormous burden of childhood illnesses, cancer is, for many, not a priority. It is reported that globally $80 \%$ of the children who develop cancer have no or poor access to cancer treatment ${ }^{1}$ but childhood cancer is often curable and every child needs care, be it curative or palliative.

In this paper we describe the children's cancer unit (called Sobo ward) in the Queen Elizabeth Central Hospital $(\mathrm{QECH})$; some of the common cancers and treatments given and their outcome. This is written with the intention to encourage early referral of children who need investigation for possible malignancy and to highlight what positive results can be achieved.

\section{The ward}

Children with cancer have been treated in QECH since 1964. In 1997 a separate paediatric oncology ward was opened and dedicated to the treatment of children with cancer. It has 17 beds. It is staffed by 5 nurses, a play lady, a social worker, 1 clinical officer, and daily attendance of a paediatrician. A paediatric surgeon visits twice weekly.

Medical treatment (drugs and investigations) is free and the hospital supplies two meals a day for each patient and one guardian. Patients come mainly from the southern region of Malawi, but some are from the North or Central regions and some have travelled from Mozambique.

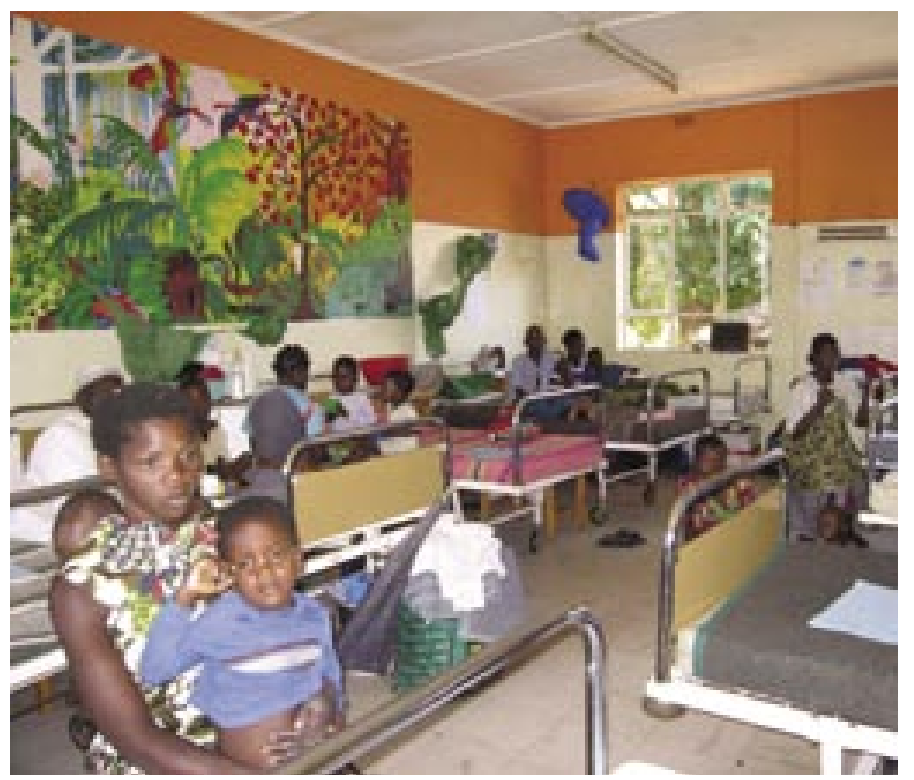

Sobo Ward, Queen Elizabeth Central Hospital Blantyre

\section{Facilities, supportive care}

Facilities have steadily improved over the years. We can do full blood counts, blood cultures, electrolytes, $\mathrm{X}$ rays, ultrasound scans, CT-scans and MRI scans. A range of chemotherapeutic drugs is available. Good supportive care is essential when giving chemotherapy to patients. These supportive care facilities are first line and second line antibiotics, antifungal therapy, topical and oral antiviral drugs, blood products, nutritional support, and adequate pain control. The chemotherapy protocols in general are less toxic than protocols used in the West to avoid treatment related morbidity and mortality.

\section{Common problems at presentation}

Children with cancer in Malawi often present late with advanced disease. Access to health care, transport problems, lack of knowledge of the disease and possible treatment all play a role. Many children remain undiagnosed. The incidence of cancer in children $<15$ years in Europe is around 140 per million children. ${ }^{2}$ Transferring this figure to Malawi with a population of 6.5 million children below the age of 15 years would mean that we would expect an incidence of around 900 cases of childhood cancer per year in Malawi.

\section{Malnutrition - nutritional support}

We found that $55 \%$ (70 of 128) of children with cancer admitted to the Queen Elizabeth Central Hospital are acutely malnourished at admission. This is caused by the high prevalence of undernutrition in Malawi, combined with the often delayed presentation with a malignant disease. Weight for height is not a good assessment of malnutrition in these children as large (often abdominal) masses mask the loss of body weight. In our study, weight for height detected malnutrition in only $17.2 \%$ (22 of 128) of children yet $55 \%$ were malnourished using other criteria such as MUAC (mid upper arm circumference), skinfold thickness or arm muscle area AMA. ${ }^{3}$ Nutritional support is essential during treatment and we provide chiponde, a locally made ready to use therapeutic peanut butter-based food which is protein and energy rich. Naso gastric tube feeding is rarely practised as parents are reluctant to accept it.

\section{Social support - failure to complete treatment}

Failure to complete paediatric cancer treatment is a common problem in developing countries. ${ }^{4}$ The treatment may take a few weeks, sometimes even months. During this time the child and his/her guardian usually stay in the hospital. Financial costs (transport, food during their stay in the hospital) are important concerns for parents. The absence from home (work on the field, income generating activities) is another concern.

Parents in Malawi are highly motivated to continue with treatment if they think that it will cure their child. The diagnosis of cancer is usually made on admission to hospital 
and causes fear of recurrence and death. Guardians are reluctant to ask health personnel questions about recurrence and outcome. They worry that taking frequent blood samples will weaken their child. The side effects of the chemotherapy, such as vomiting, are seen as a proof of efficacy. ${ }^{5}$

A play lady is present on the ward every working day of the week. She has some toys and drawing material. This is greatly appreciated by the children as they are away from home and their play mates for a long time.

\section{Patients}

Currently about 160 new patients are admitted each year. Burkitt's lymphoma is the most common cancer in malaria endemic parts of Africa $^{6}$ and more than half of the patients have Burkitt's lymphoma. About $10-15$ a year have Wilms' tumour, 10 have other lymphomas and approximately 20 have AIDS related Kaposi's sarcoma. ${ }^{3}$ Patients with retinoblastoma are seen first by eye specialists. Patients with osteosarcoma are often first seen by orthopaedic surgeons. (Table 1)

Table I. Clinical diagnoses of children admitted to Sobo ward QECH January 1st 2007-January 1st $2008 \quad(n=136)$

\begin{tabular}{|l|l|l|l|}
\hline Clinical diagnosis & Number & $\%$ of total & $\begin{array}{l}\text { FNA } \\
\text { confirmed } \\
\text { (Number) }\end{array}$ \\
\hline Burkitt's lymphoma & 82 & 60.2 & 52 \\
\hline Lymphoma (not BL) & 10 & 7.3 & 2 \\
\hline Wilms' Tumour & 11 & 8 & 6 \\
\hline Neuroblastoma & 6 & 4.4 & $0^{*}$ \\
\hline Rhabdomyosarcoma & 4 & 2.9 & 2 \\
\hline Germ cell tumour & 2 & 1.4 & 1 \\
\hline Hepatocellular carcinoma & 3 & 2.2 & 1 \\
\hline Osteosarcoma & 4 & 2.9 & 2 \\
\hline Leukaemia & 1 & 0.7 & 0 \\
\hline Other (or unknown) & 7 & 5.1 & 3 \\
\hline Brain tumour & 2 & 1.4 & 0 \\
\hline Retinoblastoma & 4 & 2.9 & 4 \\
\hline
\end{tabular}

FNA - fine need aspirates

* Two fine needle aspirates (FNAs) showed small blue round cells.

\section{Burkitt's lymphoma}

Burkitt's lymphoma (BL) is a very rapidly growing tumour. Children commonly present with a mass in the jaw, orbital socket or abdomen. They may present with weakness in the legs, paraplegia and urinary incontinence, caused by a tumour involving or compressing the spinal cord. ${ }^{7}$ This is a condition which needs urgent treatment. With treatment, we often see complete recovery of the neurological signs.

In well resourced countries patients with BL have a 1 year survival of $>90 \%$ when treated with very intense protocols requiring intense supportive care. ${ }^{8}$ Over the last 10 years a local protocol has been developed for the treatment of patients with Burkitt's Lymphoma. The previous protocol, modified from a protocol (called the LMB protocol) showed an unacceptably high mortality rate related to treatment. Eleven of $42(26 \%)$ children died of treatment related toxicity, such as sepsis with neutropenia. ${ }^{8}$ The protocol has been modified over several years and is now a shorter, cheaper protocol with limited toxicity. ${ }^{8}$

The treatment for children with Burkitt's lymphoma (all stages) consists of intravenous cyclophosphamide $40 \mathrm{mg} /$ $\mathrm{kg}$ on day 1 and oral cyclophosphamide $60 \mathrm{mg} / \mathrm{kg}$ on day 8,18 and 28 . Intrathecal hydrocortisone $(12.5 \mathrm{mg})$ and methotrexate $(12.5 \mathrm{mg})$ are also given at each treatment cycle. The 1 year event free survival of this protocol is $48 \%$ , The treatment related mortality is around $5 \%$. ${ }^{9}$

Rescue treatment (3 weekly courses of high dose cyclophosphamide $(60 \mathrm{mg} / \mathrm{kg})$ and vincristine $(1.5 \mathrm{mg} / \mathrm{m}$ 2IV) for 28 cases of relapse $(n=20)$ or primary resistance $(n=8)$ led to a disease free survival of $>$ one year (406-712 days) in a further one third of all relapsed cases. ${ }^{\text {? }}$

\section{Wilms tumour}

Worldwide Wilms tumour has an incidence of around 8 per million children below the age of 15 years. ${ }^{10,11}$ The common presentation is a child of about three years old, with a painless, firm mass in the flank.

In QECH, about 15 new patients with Wilms tumour per year are seen. Many children present with very large tumours weighing over $2 \mathrm{kgs}$ with local or distant spread. Patients with Wilms tumour in the West have a 5 year disease free survival greater than $80 \%{ }^{12}$ A previous retrospective review in the QECH showed a survival of $20-50 \%$ with $30 \%$ lost to follow up. ${ }^{13}$ This was when only vincristine was available for preoperative chemotherapy. We now have all the drugs that are used in protocols designed by SIOP (International Society of Paediatric Oncology) and use these protocols which have been modified to the situation in Malawi. The preoperative treatment for localized disease is four weeks of vincristine and actinomycin; for metastatic disease (usually chest, sometimes liver) doxorubicin is added and the treatment period prolonged to 6 weeks. Postoperative treatment depends on the stage and grade of the tumour at surgery and is usually a total of 26 weeks, with intervals of 4 weeks when patients can go home.

\section{A boy with Wilm's tumour}

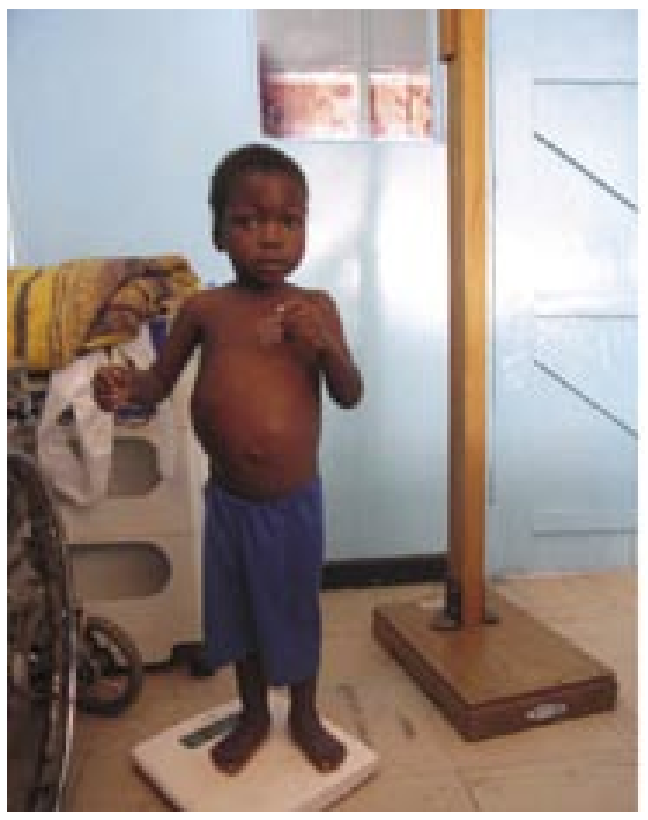


We have yet to see if this treatment has lead to improved disease free survival.

\section{Kaposi's sarcoma}

AIDS related Kaposi's sarcoma is a common disease in Sub Saharan Africa, both in adults and in children. ${ }^{14}$ It used to be a very rare cause of generalised lymphadenopathy in young children before the HIV epidemic emerged. In 10 years from 1967-76 only 11 cases were reported in Malawi. ${ }^{15,16}$ The incidence has increased over the last $10-20$ years with the rise of prevalence of HIV infected children. ${ }^{17,18} \mathrm{KS}$ can clinically present in several ways. It commonly causes skin lesions on the feet, legs, face (nose) and genitalia. It can cause painful and functionally disturbing lymphoedema especially in the lower extremities, but also in the face and genitalia. KS often presents with lesions in the oral cavity which can be painful and hamper food intake. It may cause gastrointestinal - or (life threatening) pulmonary disease.${ }^{19}$ Kaposi's sarcoma is a stage IV AIDS defining disease and qualifies a child in Malawi to start anti retroviral therapy (ART). ARTs are the best treatment for KS but in extensive, symptomatic KS additional treatment is needed. We have been giving children injections of vincristine (weekly for 3 weeks then fortnightly for 3 courses) as additional treatment. We have shown that thalidomide is an effective palliative therapy in widespread $\mathrm{KS} .{ }^{20}$ We are planning to compare different treatment options in the future to be assess how best to treat these children with minimal toxicity and improved quality of life.

\section{Other cancers}

Acute leukaemia is the most common cancer to be diagnosed in well resourced countries and yet it is rarely diagnosed in Malawi. This is probably because the symptoms (anaemia, fever) resemble the clinical picture of malaria. Besides this, when left untreated patients with leukaemia may die within a relatively shortperiod of time (of weeksora few months). Many other children present with other tumours such as Hodgkin's lymphoma, non Hodgkin's non Burkitt's B cell lymphomas, retinoblastoma, osteosarcoma, rhabdomyosarcoma, hepatocellular carcinoma and neuroblastoma. ${ }^{17}$ Some are curable; many require symptomatic care and management.

\section{Palliative care}

Even if not all children can be cured it is still important that we give adequate and holistic care to all children with cancer and to their families. There is much that can be done to give symptomatic relief, emotional and psychological and spiritual support. Many of the children with non-Wilms solid tumours presenting with advanced stage disease have a poor prognosis. Good palliative care including adequate pain control is essential. The Umodzi paediatric palliative care team do rounds on the ward every day and home visits when necessary. A description of their activities appears elsewhere in this journal.

\section{Conclusion}

Malignant disease in children can be treated. BL can be cured in $60 \%$ of cases. We anticipate improved long term survival with the Wilm's tumour protocol now in use in the QECH. All children with suspected cancer need evaluation, diagnosis and a proper treatment and management plan which is best undertaken by an experienced team. Delay in referral and treatment are detrimental to the child and the family. We have shown that for the most common tumours found in Malawian children good results can be obtained with simple and safe protocols.

\section{Acknowledgments}

We would like to thank all the Sobo staff for their care of the children and to express our humble admiration for all the children and their families who display great patience, good humour and kindness throughout their enforced hospital stays.

\section{References}

1. UNICEF. The state of the world's children 2008

2. Steliarova-Foucher E, Stiller C, Kaatsch P. Geographical patterns and time trends of cancer incidence and survival among children and adolescents in Europe since the 1970s (the Accis project): an epidemiological study. Lancet 2004;364:2097-1053

3. Israels T, Chirambo C, Caron HN, Molyneux EM, Nutritional status at admission of children with cancer in Malawi. Pediatr Blood Cancer (2008) published online Jul 30.

4. MacDougall LG, Wilson TD, Cohn R, Shuenyane EN, McElligott SE Compliance with chemotherapy in childhood leukaemia in Africa. SAMJ 1989;75(10):481-4

5. Israëls T, Chirambo C, Caron H, de Kraker J, Molyneux EM, Reis R. The guardians' perspective on paediatric cancer treatment in Malawi and factors affecting adherence. Pediatr Blood and Cancer 2008:Epub Jul 30

6. Stewart BW, Kleihues P. World Cancer Report Page 237. World Health Organisation International. Published by IARC 2003 Lyon, France.

7. Parkin DM, Ferlay J, Hamdi-Cherif M, Siton F, Thomas JO, Wablinga H, Whelan SL. Cancer in Africa: Epidemiology and Prevention.Chapter 4.10 pages 325-328. IARC Scientific Publication No 153. IARC Press 2003. Lyon France.

8. Hesseling PB, Broadhead R, Mansvelt E, Louw M, Wessels G, Borgstein E, Schneider J, Molyneux E. The 2000 Burkitt Lymphoma trial in Malawi Pediatr Blood Cancer. 2005. 44(3):245-50

9. Hesseling PB , Molyneux E, Kamiza S, Broadhead R. Rescue chemotherapy for patients with resistant or relapsed endemic Burkitt lymphoma Trop Med Int Health. 2008;102(6):602-607

10. Stiller CA, Parkin DM. Geographic and ethnic variations in the incidence of childhood cancer. Br Med Bull 199652 (4): 682-703

11. Parkin DM, Ferlay J, Hamdi-Cherif M, Siton F, Thomas JO, Wablinga H, Whelan SL. Cancer in Africa: Epidemiology and Prevention.Chapter page 389. IARC Scientific Publication No 153. IARC Press 2003. Lyon France.

12. Kaste SC, Dome JS , Babyn PS, Graf NM et al. Wilms tumour: prognostic factors, staging therapy and late effects. Pediatr Radiol 200838 (1) 2-17

13. Wilde J, Lameris W, Borgstein E. Personal communication

14. Dedicoat M, Newton R. Review of the distribution of Kaposi's sarcomaassociated herpes virus (KSHV) in Africa in relation to the incidence of Kaposi's sarcoma. British Journal of Cancer. $2003 ; 88: 1-3$

15. Molyneux EM. Childhood Malignancies in Malawi 1967 - 76. East African Med J 1979;56:15 - 21.

16. Kaposi Cancer treatment - National Cancer Institute. www.cancer.gov/ cancertopics/pdg/treatment/Kaposis/Patient/Page3

17. Sinfield R, Molyneux EM, Banda K, Broadhead RL, Borgstien E, Mkandawire NK, HB Nkume, Liomba G. The prevalence, presentation of paediatric malignancies in Blantyre Malawi in the HIV era, 1998 -2003 Pediatric Blood and Cancer 2007;48(5):515-20.

18. Molyneux E. HIV-AIDS and cancer in children - double trouble. Medical and Paediatric Oncology. 2003; 41, (4): 0041, 267.

19. Detailed Guide: Kaposi's Sarcoma. What is Kaposi Sarcoma? American Cancer Society. http://www.cancer.org/docroot/cri/content/cri_2_4_ 1x_what_is_kaposis_sarcoma_21 asp

20. Hodgson T, Kondowe W, Molyneux E, Mhango T, Sinfield R, Packer S, Mbopi-Keou F, Teo C-G, Scully C, Porter S. Thalidomide for palliation of Kaposi's Sarcoma in Malawian Children. Abstract. SIOP/PAPSA 6th Africa Continental Meeting, Mangochi Malawi. May 3-6th 2004 Pediatric Blood and Cancer;2005;44:292 